\title{
Using WebMO to Investigate Fluorescence in the Ingredients of Energy Drinks
}

\author{
Mark Smith \\ Arthur CUSD \#305 \\ 301 E. Columbia St. \\ Arthur, IL 61911 \\ 001-217-543-2146 \\ msmith@arthur.k12.il.us
}

\author{
Emily Chrisman \\ Pleasant Plains CUSD \#8 \\ 500 N. Cartwright St. \\ Pleasant Plains, IL \\ 001-217-626-1044 \\ echrisman@ppcusd8.org
}

\author{
Patty Page \\ PBL CUSD \#10 \\ 600 Orleans St. \\ Paxton, IL 60957 \\ 001-217- 379-3314 \\ pageps@earthlink.net
}

\author{
Kendra Carroll \\ Shiloh CUSD \#1 \\ $21751 \mathrm{~N} 575$ th St. \\ Hume, IL 61932 \\ 001-217-887-2365 \\ kcarroll@ista-il.org
}

\begin{abstract}
With computers becoming more powerful tools, computational modeling can be introduced gradually to secondary students allowing them to visualize complex topics and gather data in the different scientific fields. In this study, students from four rural high schools used computational tools to investigate attributes of the ingredients that might cause fluorescence in energy drinks. In the activity, students used the computational tools of WebMO to model several ingredients in energy drinks and gather data on them, such as molecular geometry and ultraviolet-visible absorption spectra (UV-Vis spectra). Using the data they collected, students analyzed and compared their ingredient molecules and then compared them to molecules that are known to fluoresce to determine any patterns. After students participated in this activity, data from testing suggest they were more aware of fluorescence, but not more aware of how to read an UV-Vis spectrum.
\end{abstract}

\section{Categories and Subject Descriptors}

K. Computing Mileux, K..3 Computer and Education, K..3.1 Computers uses in education

\section{General Terms}

Measurement, Performance, Design, Human factor, Experimentation, Theory

\section{Keywords}

Fluorescence, Molecular Geometry, WebMO, Molecular Shape, Energy Drink, Computational Science, Education, Chemistry, High School, Absorption Spectra, UV/Visible

\section{INTRODUCTION}

Students today have changed greatly in the methods that they use to seek and obtain information in their lives. Teachers who are aware of this change can adapt their methods to better serve their students. These adaptations should enhance students' educational experience and increase their comprehension of the subjects being taught. An important difference between students today and those in the past is their use of desktop, laptop, netbook, and tablet personal computers (PCs). They have become well versed in the use of computers for information gathering and entertainment. These computers are capable of running programs that can create highly accurate simulations and models in chemistry. In an effort to determine the impact of computer simulations and computational models on student comprehension in chemistry, the National Science Foundation (NSF) is funding the Institute for Chemistry Literacy through Computational Science (ICLCS), a five year program hosted by the University of Illinois at UrbanaChampaign (S.Sendlinger, personal communication, ICLCS workshop 2008). The ICLCS program has given rural chemistry teachers from all over the state of Illinois, including over ninety school districts, the opportunity to come together and be introduced to some computational tools and trained in their use. After the training, these teachers were asked to create an "Ice Cube," which is a small unit that could take one to two class periods to complete and uses computational tools. The teachers were asked to test their "Ice Cubes" in their classes and gauge the effectiveness by a pre/post test system as well as qualitative observations. The use of computational tools in the classroom is believed to help students understand some of the more abstract and hard to visualize concepts in chemistry. It does this by giving students the ability to manipulate visual models of the conceptual aspects of chemistry. Manipulations in these computational tools allow students to "see" and experiment with molecules and their behavior in a more concrete way.

The "Ice Cube" used in this study used computational tools to introduce fluorescence to the students. It incorporated real-world scenarios, inquiry, research, complicated modeling and molecular computations to further the student's knowledge of this material. Students were shown the fluorescence of a common energy drink. This started the inquiry process of why this happens. The students researched the ingredients and fluorescence to further their 
understanding. The teachers in leading this project introduced the computational tool WebMO to help the students further their investigation.

\section{METHODS}

\subsection{Instructional Design}

This particular "Ice Cube" was envisioned as a part B in a three part plan where A is introductory material such as Molecular Shape, Bonding, or Orbitals/Energy levels, etc. Part C would be a springboard to transition into another area of study using information or practices used in the "Ice Cube". In this study, to gain the attention of the students, they were given the illustration of common energy drinks glowing under a black light. This illustration was designed to start the students on an inquiry investigation on the topic of fluorescence. The students were encouraged to collect data and hypothesize why some molecules fluoresce. They were guided using positive questioning techniques to explore the molecular geometry and the ultraviolet visible absorption spectra to collect data that may lead to an explanation of what causes this phenomenon. In each classroom, students were split into four teams and investigated two different common ingredients including citric acid, vitamin B6, vitamin $\mathrm{C}$, caffeine, vitamin B2, glucose, fructose, and vitamin A. Students used WebMO (http://webmo.ncsa.uiuc.edu), a computational program that can be used to calculate molecular geometries and ultraviolet visible absorption spectra, in addition to many other parameters. The program uses a powerful set of computations run on supercomputer-based servers to calculate the geometry, spectra, bond energies, and other data after the user inputs the basic shape of a molecule. WebMO's optimized geometry and computed UV-Vis spectra of the common ingredients of energy drinks were analyzed by students to see if they could find any indications that the material might fluoresce.

The total sample of ninety students had similar experiences in three of the classrooms, with 24 of the students being in the control group. The Chemistry I students were mainly in tenth grade, a few students were in the eleventh and twelfth grades. The Anatomy and Physiology class mainly consisted of eleventh and twelfth grade students. These students were first administered the pretest with no instruction. The pretest included questions on fluorescence, basic chemical symbols, graph reading, and emission spectra. The questions on the pretest and post test were:

1. Define fluorescence

2. What causes fluorescence?

3. What does the double line in the figure represent? ${ }_{\mathrm{OH}}$
a. double strength bond
b. double bond
c. two carbon atoms present
d. more hydrogen present

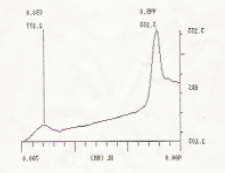

4. Does the emission spectrum above show visible light?
a. Yes
b. No
c. Not enough information

5. Using the same graph, what energy value has the greatest intensity?

$\begin{array}{ll}\text { d. } & \text { a. } 658 . \mathrm{nm} \\ \text { e. } & \text { b. } 445 \mathrm{~nm} \\ \text { f. } & \text { c. } 3.233 \\ \text { g. } & \text { d. } 2.227\end{array}$

The students then completed the "Ice Cube." Students were broken into small groups of two or three and subsequently into four different teams. The students were given the Student Instruction sheet and Student Datasheet. Once students were broken into teams, they used WebMO to build an ingredient molecule from the energy drink using a provided structural formula, as in Figure 1.

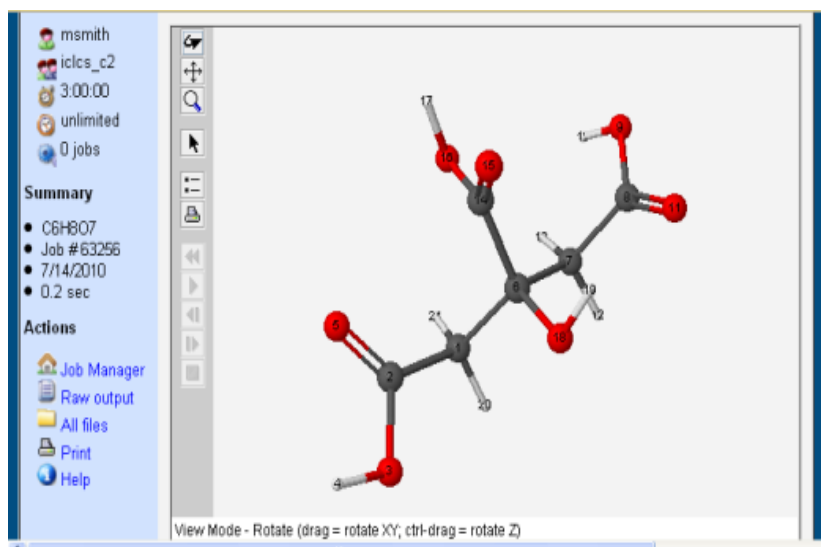

Figure 1 Citric acid built in WebMO

Students recorded the UV-Vis spectrum and structure in their data sheet. Then students imported a completed, more complex ingredient molecule into WebMO from the National Institute for Science and Technology's Chemistry WebBook website (http://webbook.nist.gov/chemistry/) (2008). The students then performed the same processes and recorded their results on the data sheet, as in Figure 2 and Figure 3. 


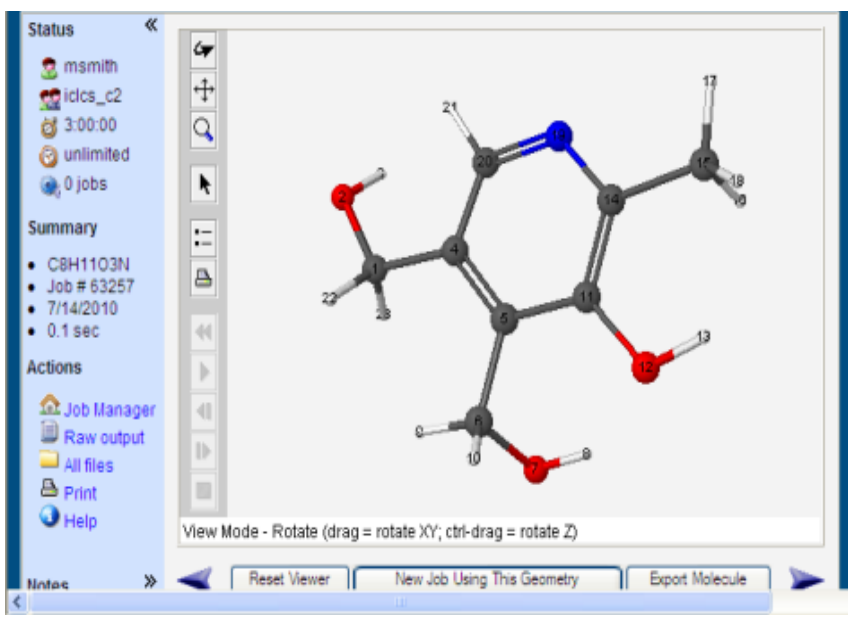

Figure 2 Vitamin B6 imported and optimized in WebMO

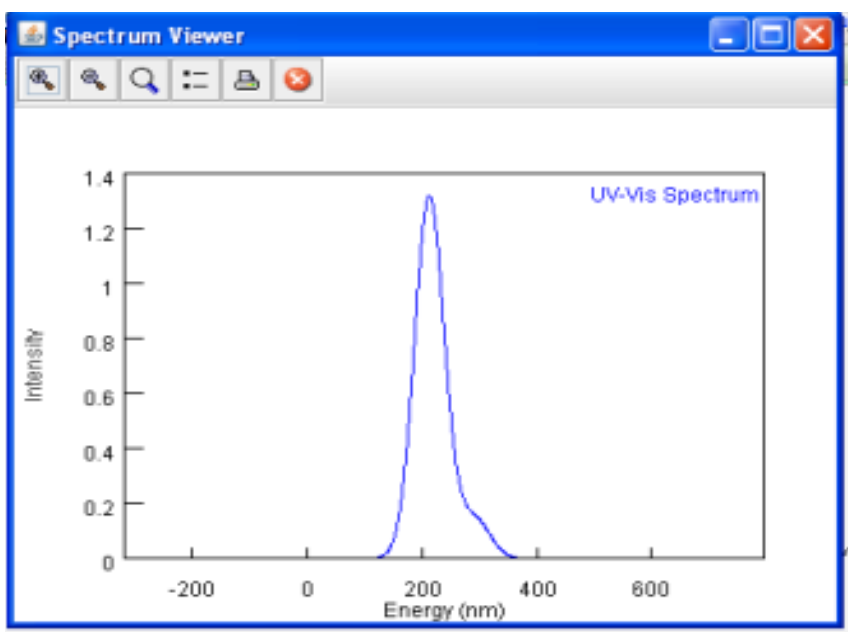

Figure 3 UV-Vis Spectrum of Vitamin B6

Following these activities, students answered a series of data analysis questions to try to determine if there were similarities between the two ingredients. The students then compared their data to the data of chemicals that were known to fluoresce and commented on whether or not they thought their ingredients would fluoresce. Finally, the class discussed the possibility of any one of these ingredients being the one that caused the fluorescence in the energy drink. The class also discussed why literature on the cause of fluorescence in energy drinks seemed to be nonexistent. After the "Ice Cube", thirty-one Chemistry I students and ten Anatomy and Physiology students received traditional instruction on fluorescence and the emission spectra. The other twenty-five students participating in this "Ice Cube" did not receive traditional instruction. In the end of the lesson, all students took the same assessment that was given in the beginning of the "Ice Cube" as the post test. The post test responses indicate that students may have greater understanding in these topics.

\subsection{Computational Tools}

After creating the model of the molecule using the editor in WebMO (http://webmo.ncsa.uius.edu ), students first ran the molecule through a geometry optimization using MOPAC/PM3 modeling parameters. This step optimized the model into its lowest energy state. The students then found the ultraviolet visible absorption spectrum using the Hartree-Fock/Other (null) modeling parameters, as in Figure 4.

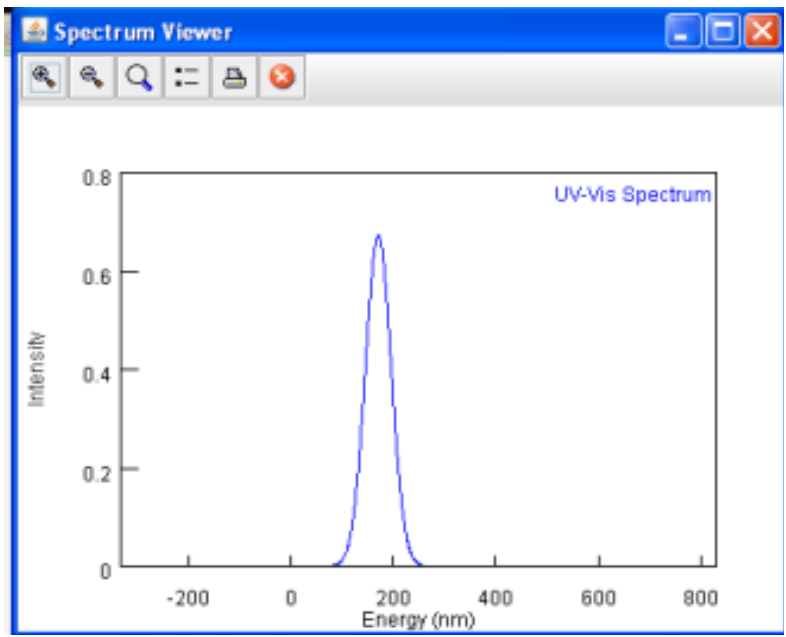

Figure 4 UV-Vis Spectrum for Citric Acid

\section{RESULTS}

The following results, in Table 1 were gained by having sixty-six students in three high school Chemistry I classes and one high school Anatomy and Physiology class participate in this activity. These classes were in three different rural Illinois schools. Of the sixty-six students, all of them took a pretest and post test for the activity and had the following results. The table also compares Chemistry I students who did receive traditional instruction after the WebMO activity versus the Chemistry I students who did not. Twenty-four Chemistry I students acted as a control and were given the pretest and post test after traditional instruction only. The data in Table 1 shows the overall improvements gained by each type of student.

The control group, receiving only traditional instruction, had a gain of 0.65 points. The Anatomy and Physiology group showed a gain of 1.7 points after using WebMO. The Chemistry I students with both WebMO ice cube and traditional instruction showed a gain of 0.55 points and the Chemistry I students with just the ice cube showed a gain of 1.12 points. The students with the WebMO experience also always had a higher post test score than the control group. This data shows that significant gains were achieved with the use of WebMO. When compared to the control group with traditional instruction only, it was found that the use of WebMO, on average, did have the most gain of understanding on the topic of fluorescence. It was also noted that the free response answers on the pretest were more indepth for the students who participated in the project and did some research on their own. The students receiving only traditional instruction had answers ranging from "no clue" on the pretest to "emission of a 
photon" when asked to define fluorescence. Although the final answer is a component of the correct answer, it was not a complete answer.

\section{Table 1 Pre/Post Test Results}

\begin{tabular}{|c|c|c|}
\hline Classes & \begin{tabular}{|l|} 
Pretest average \\
score out of 5
\end{tabular} & \begin{tabular}{|l} 
Posttest average \\
test score out of 5
\end{tabular} \\
\hline $\begin{array}{|ll|}\text { Anatomy } & \text { and } \\
\text { Physiology } & \\
10 \text { students } & \\
\end{array}$ & 1.8 & 3.5 \\
\hline $\begin{array}{|lr|}\begin{array}{l}\text { Chemistry } \\
\text { with }\end{array} & \text { students } \\
\text { instruction } & \text { traditional } \\
& \\
31 \text { students } & \\
\end{array}$ & 2.85 & 3.4 \\
\hline $\begin{array}{|lr|}\text { Chemistry } & \text { I } \\
\text { without } & \text { tradents } \\
\text { instruction } & \\
& \\
25 \text { students } & \\
\end{array}$ & 2.08 & 3.2 \\
\hline \begin{tabular}{|l} 
Chemistry I students \\
with only traditional \\
instruction
\end{tabular} & 1.25 & 1.9 \\
\hline
\end{tabular}

All students in the classes tested made improvements from their pretest to the post test scores, which may indicate an increased understanding of what fluorescence is and how it works. Students' answers on the short answer part of the assessment became more in depth and comprehensive. On the pretest, examples of their answers to the question "Define fluorescence." were; blank answers, "a molecular process", "the amount of energy to complete something", "chemical substances made from fluorine", and "I don't know". Of the twenty-five Chemistry I students, twenty-three students were unable to properly define fluorescence. Most students seemed to have no prior knowledge of what fluorescence was before the activity. The activity seemed to improve their understanding of what fluorescence is and what causes compounds to fluoresce. On the posttest, answers to the same question become more in-depth, like "the emission of light after an excited state".

\section{DISCUSSION}

Research has shown that fluorescence is best understood by looking deeper than just the geometry of a molecule. Although double bonds may be a good reason to look deeper, the possible transitions that cause fluorescence are effected by not only the arrangement of nonbonding and bonding orbitals within the molecule but also the environment such as hydrogen bonding. Moreover because energies are effected by various conditions including the orientation of the $\mathrm{p}$ orbitals, the circumstances leading to fluorescence in molecules differ depending on whether the molecule is azoaromatic, or a carbonyl, etc (Sidman, 1958). Because understanding the reasons for fluorescence are so complex students would not be able to reach any clear answer, but should be able to hypothesize some generalities.

Students were able to perform the energy drink activity easily. Most of the students had previous working knowledge of WebMO and the team structure allowed the inexperienced students to complete the lab. The directions were detailed and step-by-step preventing students from making many mistakes. Unfortunately, there was a problem in the procedure for importing molecules that was addressed in the student instructions. The student instructions failed to tell students after importing their second molecule to open the editor in WebMO and select "Cleanup; Comprehensive mechanics" to attach hydrogens where needed. This mistake caused numerous failures of their model runs for several groups, which began to frustrate some of the students until the mistake was found and corrected in the instructions. Students also had trouble building the molecules they were assigned. However, with teacher guidance they were able to rebuild the molecule quickly and complete the lab without incident. It was also observed that once one student was shown how to overcome a technical problem in WebMO, they would help others around them. This helped foster cooperative learning. Overall, the instructions were well written and allowed the activity to be completed in a timely manner.

The data sheet also had its pros and cons. Overall, the data sheet was easy for students to follow and complete. Some students needed clarification with a few of the questions; however, the data sheet was clear and concise. Unfortunately, there was a problem with student understanding in the questions that dealt with the ultraviolet visible absorption graphs. Students consistently missed questions dealing with the ultraviolet visible absorption graphs in the WebMO output and how to interpret them as evidenced in the post test. More time discussing these graphs may have furthered understanding. Also, some students answered some questions incompletely, listing only one similarity between substances. Overall, students were able to perform the activity in an acceptable time with minimal questions. The post test seems to indicate that this experience did allow students to gain knowledge of fluorescence and factors that could perhaps cause it.

In the chemistry 1 class during the post test, out of the twenty-five students, sixteen consistently missed at least one of the two questions that referred to the emission spectrum graph. Even after completing the lab, they were unable to decide if the wavelength or the intensity indicated the visibility of light. They also were unable to distinguish which part of the graph, the intensity or the wavelength, indicated the amount of energy being released. Also, in the laboratory data sheets students only recognized one similarity in either the molecular geometry or the ultraviolet visible absorption spectrum. They were unable to find multiple similarities or find similarities in both the molecular geometry and ultraviolet visible absorption spectrum. Finally, students were unable to draw the conclusion that using only ultraviolet visible absorption and molecular geometry did not give them enough information to determine if a molecule would fluoresce or not. The students tested did not understand that this is a current area of study and a definitive answer is not available. After the post test, the control group of twenty-three students had nine students correctly identify if light was visible on an emission spectra graph. None, however, could identify which part of the graph, the 
intensity or the wavelength, indicated the amount of energy being released. This showed that the project did allow for further understanding of the energy associated with the emission spectra.

To fix the problems faced in this activity some changes should be implemented. The first clear change would be to add steps in the instructions for importing molecules. We need to clarify that the students should clean-up their molecule to add hydrogen in the needed places for the proper computation to take place. The data from the students supports the idea that this "Ice Cube" is best used as a part B where A is the introductory material for the subject being studied that could include listing and identifying molecular substructures such as carbonyl, nitroso, azo, and other hetero groups as well as aza aromatic compounds or simply recognizing ring structures, double-bonds and alternating bonds. Other introductory material could include experience with optics, the electromagnetic spectrum, light intensity, emissions, wavelength and frequency. Using the data from the students, the activity should be edited to help achieve more understanding of ultraviolet visible absorption and the differences in molecular geometry (J. Sidman, 1958). There should be more introduction and discussion of the ultraviolet visible absorption, and what wavelength can be represented and the visible spectrum. Finally, students should be prompted in the data sheet to look more deeply into similarities in geometry and ultraviolet visible absorption. This activity was designed to illustrate the unknown properties of scientific discovery. Students have very little contact with the unknown in the traditional classroom setup. Very little can be done to improve the frustration of the students in an activity where the outcome or answer is unknown. This can only be eased with more exposure to this type of activity.

Ultimately this activity allowed for the students to be active participants in the scientific method and in learning the complexity of determining a cause for fluorescence. The students were asked to gather information, ask questions, submit a hypothesis, test this hypothesis and discuss the results. They were put into a situation where they were testing a problem without a clear answer. This confused most students but also gave experience in real-world science. The students gained insight on doing research when there is no clear answer. This model, when compared to the control group, showed furthered understanding of this topic and scientific reasoning.

\section{CONCLUSION}

WebMO allowed students to build complex virtual molecules that they would have difficulty building in the classroom using other modeling techniques, such as ball and stick models. Also, WebMO allowed students to use the molecule they built to find new information about each molecule. Students looked at structure, the molecular geometry as well as ultraviolet/visible absorption in hopes of understanding how these features might affect its fluorescence. From the data collected, using WebMO allowed students to gain a better understanding of the concept of fluorescence. The experiment also allowed students to participate in a lab that would otherwise require some very expensive equipment to find the spectra of the molecules or require the use of literature to find the spectra. The "Ice Cube" allowed them to be active participants in the learning of the scientific method and how complex the topic of fluorescence is. WebMO allowed students to visualize the molecules they studied instead of just looking them up in reference books. WebMO is a powerful tool that allows students to investigate electron density, infrared spectra, NMR and other data. With today's students being more and more technologically savvy, WebMO can be used to help introduce complex topics that are almost impossible to visualize in real life.

Ultimately, this activity did succeed in encouraging and fostering scientific inquiry in the students. The students gained experience in taking real-world occurrences and applying the scientific method to understand the mechanisms behind observations. This activity also allowed the students to apply learned topics in chemistry to their everyday experiences. This activity did improve student understanding. Students, when compared to the control group, had a more in-depth knowledge of fluorescence, molecular structure, graph reading after participating in this activity. Further improvements will make this an even more successful way of introducing these topics.

\section{ACKNOWLEDGEMENTS}

We express our sincere appreciation to the ICLCS staff and members for their support and encouragement on this paper and the development of this "Ice Cube". We also would like to thank the University of Illinois for the use of their facilities and supercomputers and NSF for their funding of this program.

\section{REFERENCES}

[1] NIST Chemistry WebBook (2008). Molecules used for importing. Retrieved from: http://webbook.nist.gov/chemistry/

[2] Sidman, Jerome W.; Electronic Transitions Due to Nonbonding Electrons in Carbonyl, Aza-aromatic, and Other Compounds; Dept. of Chemistry, Cornell University, Ithaca, New York March 1, 1958 